\title{
The Wave Based Method: an overview of 15 years of research
}

\author{
Elke Deckers*, Onur Atak, Laurens Coox, Roberto D’Amico, Hendrik Devriendt, Stijn Jonckheere, Kunmo Koo, \\ Bert Pluymers, Dirk Vandepitte, Wim Desmet \\ KU Leuven - Dept. of Mechanical Engineering, Celestijnenlaan 300B - box 2420, 3001 Heverlee (Leuven), Belgium
}

\begin{abstract}
The Wave Based Method is a deterministic prediction technique to solve steady-state dynamic problems and is developed to overcome some of the frequency limitations imposed by element-based prediction techniques. The method belongs to the family of indirect Trefftz approaches and uses a weighted sum of so-called wave functions, which are exact solutions of the governing partial differential equations, to approximate the dynamic field variables. By minimising the errors on boundary and interface conditions, a system of equations is obtained which can be solved for the unknown contribution factors of each wave function. As a result, the system of equations is smaller and a higher convergence rate and lower computational loads are obtained as compared to conventional prediction techniques. On the other hand, the method shows its full efficiency for rather moderately complex geometries. As a result, various enhancements have been made to the method through the years, in order to extend the applicability of the Wave Based Method. This paper gives an overview of the current state of the art of the Wave Based Method, elaborating on the modelling procedure, a comparison of the properties of the Wave Based Method and element-based prediction techniques, application areas, extensions to the method such as hybrid and multi-level approaches and the most recent developments.
\end{abstract}

Keywords: acoustics, structural dynamics, Biot theory, Wave Based Method, Trefftz approach, multi-level, hybrid

\section{Introduction}

The modelling of the vibro-acoustic behaviour of physical systems is far from being trivial. In a general coupled vibro-acoustic system, in which structural, poroelastic and acoustic components mutually interact with each other, the system behaviour is typically determined by the coupled steady-state response of each of the components. In an ideal setting, the design engineer would have a modelling tool at his disposal, that would allow calculations over the whole frequency range of interest, which typically runs up to $20 \mathrm{kHz}$. In real life, however, this is not possible due to the limitations of the current Computer Aided Engineering tools at hand. Moreover, there is a significantly different response in different frequency regions. In general, three different frequency regions can be identified, which are problem dependent:

Low frequency range In the low frequency range, the characteristic length of the problem under study is smaller than or in the same order of magnitude as the dominant physical wavelengths in the dynamic response. In this frequency range, the response of the system is determined by well-separated modes and can be predicted by means of deterministic approaches. For vibro-acoustic problems, element-based techniques, such as the Finite Element Method (FEM) [1] and the Boundary Element Method (BEM) [2] are most commonly applied. Element-based approaches divide the problem domain or its boundary into a large number of small elements. Inside these elements, the field variables are approximated using simple, often polynomial functions. As wavelenghts shorten with increasing frequency, the element sizes also need to decrease to diminish the effect of

\footnotetext{
*Corresponding author. Tel.:+32 163224 80; fax: +32 163229 87; http://www.mech.kuleuven.be/ E-mail address: Elke.Deckers@mech.kuleuven.be
} 
interpolation and pollution errors $[3,4,5]$. As a consequence, the number of degrees of freedom (DOFs) increases, as does the size of the system matrices, limiting the practical use of element-based approaches to low frequency applications.

High frequency range When the characteristic length of the problem under study is much larger than the dominant physical wavelengths in the dynamic response, the considered problem is situated in the high frequency range. Typically, the modal density and modal overlap are high and the system is very sensitive to small variations in, for instance, material properties and geometrical details. As small variabilities are inevitable in real life applications, the response of one nominal system loses its meaning. As a result, the ensemble averaged response of a number of realisations is of interest together with its variance. In this frequency range, statistical techniques are applied; for instance the Statistical Energy Analysis (SEA) [6] is often used for vibro-acoustic analysis. The SEA divides the problem domain into a small number of subsystems in which a spatially averaged estimate of the energy level is obtained. SEA is computationally not very demanding, but relies on a number of assumptions, such as the diffusivity of the field and uncorrelated waves. Since these assumptions are only met above a certain frequency limit, the method is restricted to the high frequency range.

Mid frequency range In between the low and the high frequency range, a frequency band exists for which currently no mature and adequate prediction techniques are available. However, for many applications, this mid frequency gap coincides with the frequency range where the human hearing is highly sensitive. Therefore, solutions are sought to bridge (part of) this gap, and can be categorised according to three classes of approaches:

- Extending the frequency range of the deterministic approaches. Optimised solvers [7], domain decomposition methods [8] and multipole methods [9, 10] can be applied to the element-based approaches, knowledge of the dynamic problem can be incorporated [11, 12], etc. Trefftz approaches [13], which use exact solutions of the governing differential equation(s) to describe the field variables, can also be considered in this category.

- Extending the statistical methods towards lower frequencies. Approaches belonging to this category try, for instance, to get a better estimation of the SEA parameters [14] or try to relax on the assumptions imposed by SEA such that the modal energies in subsystems do not have to be similar [15].

- Combining deterministic and statistical approaches. This category of methods tries to combine the best of both procedures, such that a system which consists of combined stiff, deterministic components and flexible, statistical components can be tackled. The hybrid FE/SEA, which describes fully deterministic components via FE and highly random components by SEA, is the prime example [16].

The Wave Based Method (WBM) in its pure form [17], belongs to the family of Trefftz approaches [13] and forms the focus of this paper. It belongs to the first class of methods defined above, being an efficient alternative deterministic technique, using exact solutions of the governing equations to approximate the field variables. As such, it embeds a priori known information about the dynamic field in the expansion set, to speed up convergence. However, a priori known information about the solution field can also be embedded in element based prediction techniques. Consequently, before moving to the details of the WBM, it is useful to briefly position it among enrichments to element based prediction techniques and among other Trefftz approaches. A more complete and in depth discussion can e.g. be found in $[18,19,20]$ :

Enrichment of FE methods: The numerical pollution errors in FE schemes mainly originate from the use of simple polynomial shape functions to describe the field variables. Therefore, a commonly applied approach is to enrich the field variable approximation using a priori known information of the studied problem. In this respect, a.o. multiscale and generalised FEM should be mentioned.

In the multiscale approach, the solution is supposed to consist of a coarse and a fine scale solution. The coarse part of the solution is resolved using the standard polynomial shape functions, while the fine scale represents the oscillating nature of the dynamic solution which is resolved using an additional basis containing problemspecific functions. In for instance the Residual-Free Bubble method [21] the functions describing the fine scale do not contribute to the residuals of the coarse scale, such that they can be added to the solution, although their calculation is an expensive process. Farhat et al. $[11,22]$ propose the use of different propagating and evanescent 
waves to model the fine scale. Continuity between different elements is not longer inherently guaranteed, but is enforced using Lagrangian multipliers, explaining the name 'Discontinuous Enrichment Method' (DEM). The contribution factors of the enrichment functions are condensed out at element level, yielding a global system for the coarse-scale FE DOFs and the Lagrange multipliers. The DEM is capable of obtaining the same prediction accuracy as the classical FEM of the same order $p$ using approximately an order of magnitude less degrees of freedom. If the enrichment functions in a DEM formulation are chosen to be a good basis of the dynamic problem under study, the continuous polynomial field can be discarded, leading to a Discontinuous Galerkin (DG) method [23]. A system of equations in the Lagrange multipliers is obtained. The DG forms the foundation of several Trefftz methods, being discussed later.

In generalised FE methods [24], also called Partition of Unity FEM (PUFEM), the polynomial shape functions, which form a partition of unity, are multiplied with locally defined basis functions containing a priori information of the dynamic field. Problem specific enrichment functions, such as plane waves, harmonic polynomials,... are used. For simple academic examples, this method exhibits a superior modelling efficiency compared to the stabilised FE methods, but the introduction of highly oscillatory enrichments typically results in difficult model construction and ill-conditioned systems of equations. For a same accuracy as the DEM, the conditioning of the PUFEM is worse.

Enrichment of BE methods: In a similar fashion as PUFEM, a Wave Boundary Element Method (WBEM) can be constructed [25] by multiplying the polynomial BE shape functions with plane waves. The method incorporates the underlying wave behaviour of the solution fields in the numerical scheme. The application of the WBEM approach significantly increases the prediction accuracy but like PUFEM, the method suffers from ill-conditioning of the system matrices when a large number of enrichment functions is introduced. Some recent improvements allow the method to cope with complex geometries while avoiding serious ill-conditionied system matrices.

Trefftz Methods: Trefftz [13] proposed and alternative method for the Rayleigh-Ritz approach embedding more of the a priori known information of the physics implicitly into the numerical model. This is done by selecting a set of basis function which fulfill the governing partial differential equations describing the problem and which is Tcomplete. In general, these methods can be classified as direct or indirect. Direct Trefftz approaches discretise the boundary of the problem domain and express the boundary variables in terms of a T-complete function set. The degrees of freedom of the resulting system of equations are physical quantities on the boundary of the considered problem domain. The use of indirect Trefftz approaches is, however, much more wide-spread. In this case the field variables inside the problem domain are approximated using an expansion of Trefftz functions, which exactly fulfill the governing equations, but violate the boundary conditions. The weighting functions have no direct physical meaning and a post-processing step is needed to obtain the results inside the domain. Indirect Trefftz methods mainly differ in the selection of the Trefftz functions and how the weighting functions are obtained (e.g. in a variational, collocational, least-squares or weighted residual formulation). As mentioned before, a complete overview of possible Trefftz approaches does not fit in the scope of this paper, but it is worth mentioning the different approaches that are being followed.

The Equivalent Source Method, also referred to as the Method of Fundamental Solutions, the Boundary Knot Method,... describes the solution inside a problem domain using a distribution of sources outside the problem domain, e.g. see [26, 27, 28]. Many other Trefftz approaches apply a partitioning of the problem domain into elements. In this extent the subdivision into 'enhanced element approaches' and Trefftz approaches is not always very clear. The DG [23], as mentioned above, serves as a framework to derive Trefftz methods. In each element a suitable set of Trefftz functions is used. The Ultra Weak Variational Formulation (UWVF) [29] and the Hybrid Trefftz Finite Element Method (HTFEM) [30] both use plane wave basis functions, but use a weak variational approach, leading to impedance-like interelement conditions and Lagrange multipliers, respectively, to couple the interface variables.

Other Trefftz methods also omit the interface variables and apply a direct coupling between adjacent elements, as is for instance implemented in the Least-Squares T-elements (LST). The Variational Theory of Complex Rays (VTCR) [31] also belongs to the class of directly coupled domain based indirect Trefftz approaches and uses another variational formulation. It applies complex rays to describe the dynamic field, which involve two scales: a slowly varying scale and a rapidly varying scale. Only the slowly varying scale is discretised and the 
rapidly varying scale is taken into account analytically. Recently, Kovalevsky [32] proposed a truncated Fourier series to discretise the amplitudes, resulting in a smoother and more predictable convergence.

The WBM is designed for solving steady-state dynamic problems, governed by a (set of) Helmholtz equation(s). It can be applied to bounded as well as (semi-)unbounded problem domains. It belongs to the category of indirect Trefftz approaches; a post-processing step is required to determine the field variables and derivative quantities in the points of interest. The WBM applies a direct coupling approach: a weighted residual formulation is used to enforce boundary conditions and interdomain continuity conditions, such that no additional variables are introduced at the interfaces. Contrarily to element-based approaches, which divide the problem domain or its boundary into a large number of small elements, the WBM partitions the problem domain in a small number of large subdomains. Within the subdomains, the WBM approximates the field variables by a weighted sum of so-called wave functions, each of which is an exact solution of the governing differential equation(s). These wave functions comprise propagating as well as evanescent waves. The wave numbers of these waves depend on the subdomain dimensions. Model refinement is done by increasing the number of approximation functions in the subdomains, rather than increasing the number of elements.

This paper gives an overview of the current state of the art of the WBM, summarising fifteen years of research. The first section gives a mathematical description of the dynamic problems which can be solved with the WBM. Next, the four steps involved in the WB modelling procedure are discussed and are, as an illustration, applied to a 2D acoustic problem. Thereafter, the typical properties of the WB models and their strengths and weaknesses are compared to those of element-based approaches. Finally, the application areas of the WBM and its current extensions are discussed and an outlook for future WB research is given.

\section{Generalised Helmholtz problem}

Consider a general interior/exterior steady-state dynamic problem as shown in Figure 1. A time harmonic dependence $e^{j \omega T}$ is assumed, with $j$ the imaginary unit, $\omega$ the circular frequency and $T$ the time. It is assumed that the mathematical formulation of the physics inside the problem domain $\Omega$ gives rise to, or can be cast into a number of $N_{H}$ (modified) Helmholtz equations

$$
\nabla^{2} u_{m}(\mathbf{r})+k_{m}^{2} u_{m}(\mathbf{r})=\mathcal{F}_{m}(\mathbf{r}), \mathbf{r} \in \Omega, m=1, \ldots, N_{H} .
$$

In this equation, $\nabla^{2}$ is the Laplacian operator, $u_{m}(\mathbf{r})$ is the dynamic field variable of the $m^{\text {th }}$ Helmholtz equation, and $k_{m}$ is the physical wave number of the $m^{t h}$ Helmholtz equation, which is determined by the physical properties of the medium inside the problem domain $\Omega$. $\mathcal{F}_{m}(\mathbf{r})$ represents non-homogeneous forcing terms. The vector $\mathbf{u}(\mathbf{r})=$ $\left[u_{1}(\mathbf{r}), \ldots, u_{N_{H}}(\mathbf{r})\right]^{T}$, contains the $N_{H}$ considered dynamic field variables.

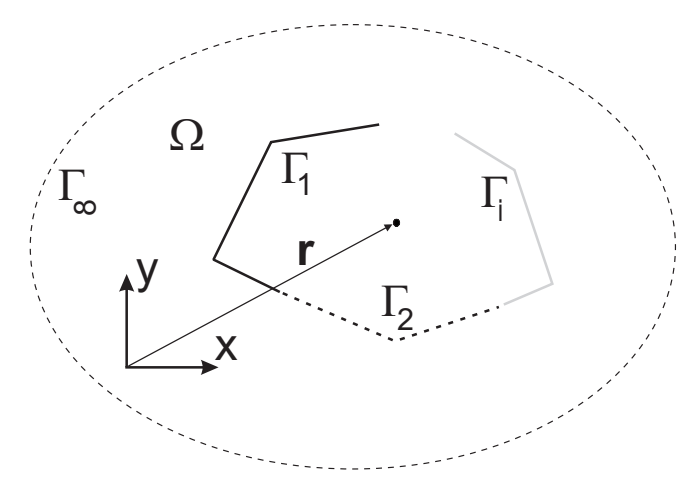

Figure 1: General steady-state dynamic problem description.

The problem boundary $\Gamma=\partial \Omega$ consists of two parts in case the problem domain is unbounded: the finite part of the boundary, $\Gamma_{b}$, and the boundary at infinity, $\Gamma_{\infty}$. For a bounded domain, obviously, only $\Gamma_{b}$ has to be considered. The finite part of the boundary can be divided into non-overlapping parts: $\Gamma_{b}=\bigcup_{i} \Gamma_{i}$, on which different boundary 
conditions can be imposed. On each point of the boundary, $N_{H}$ boundary conditions need to be defined to obtain a well-posed problem. The set of $N_{H}$ boundary conditions on a general boundary $\Gamma_{i}$ can be written in the general form

$$
\mathcal{B}_{i, l}(\mathbf{u}(\mathbf{r}))=\overline{\mathcal{B}}_{i, l}(\mathbf{r}), \quad \mathbf{r} \in \Gamma_{i}, \quad l=1, \ldots, N_{H},
$$

with $\mathcal{B}_{i, l}(*)$ a general boundary differential operator and $\overline{\mathcal{B}}_{i, l}(\mathbf{r})$ an imposed boundary field. Note that the subscript - $l$ denotes a counter, and not a derivation of the parameter $\bullet$ with respect to coordinate $l$. The $N_{H}$ number of field variables inside the problem domain are coupled through the boundary conditions. At the boundary at infinity $\Gamma_{\infty}$, non-reflecting boundary conditions are imposed, ensuring that the resulting wave field is purely outgoing and no energy is reflected back into the problem domain

$$
\mathcal{B}_{\infty, l}(\mathbf{u}(\mathbf{r}))=0, \quad \mathbf{r} \in \Gamma_{\infty}, \quad l=1, \ldots, N_{H} .
$$

The Helmholtz equation(s) (1) together with the imposed boundary conditions (2), and (3) for an unbounded problem, define unique field variables $u_{m}(\mathbf{r})$. Once these field variables are known, derived quantities can be obtained.

As an example throughout this paper, a 2D acoustic problem is considered. The fluid is characterised by its ambient fluid density $\rho$ and its speed of sound $c$. The steady-state acoustic pressure inside the problem domain is governed by the inhomogeneous Helmholtz equation [33]

$$
\nabla^{2} p(\mathbf{r})+k^{2} p(\mathbf{r})=-j \rho \omega \delta\left(\mathbf{r}, \mathbf{r}_{\mathbf{q}}\right) q .
$$

Consequently, in this case $N_{H}=1, u_{m}(\mathbf{r})=p(\mathbf{r})$, the acoustic pressure inside the domain, $k_{1}=k=\omega / c$, the acoustic wave number. In case an acoustic volume velocity source with source strength $q$ is present in the problem domain, $\mathcal{F}_{1}(\mathbf{r})=-j \rho \omega \delta\left(\mathbf{r}, \mathbf{r}_{\mathbf{q}}\right) q$, with $\delta$ the Dirac-delta function.

The finite part of the boundary can be further divided into three non-overlapping parts: $\Gamma_{b}=\Gamma_{v} \cup \Gamma_{p} \cup \Gamma_{Z}$, where the following boundary conditions apply

$$
\begin{array}{ll}
\mathbf{r} \in \Gamma_{v}: & \mathcal{L}_{v}(p(\mathbf{r}))=\bar{v}_{n}(\mathbf{r}), \\
\mathbf{r} \in \Gamma_{p}: & p(\mathbf{r})=\bar{p}(\mathbf{r}), \\
\mathbf{r} \in \Gamma_{Z}: & \mathcal{L}_{v}(p(\mathbf{r}))=\frac{p(\mathbf{r})}{\bar{Z}_{n}(\mathbf{r})},
\end{array}
$$

with $\bar{v}_{n}, \bar{p}$ and $\bar{Z}_{n}$ the imposed normal velocity, pressure and normal impedance, respectively. The local normal on the boundary is denoted by $n$ and the normal velocity operator $\mathcal{L}_{v}(\bullet)$ is defined as

$$
\mathcal{L}_{v}(\bullet)=\frac{j}{\rho \omega} \frac{\partial \bullet}{\partial n} .
$$

On the boundary at infinity $\Gamma_{\infty}$ the Sommerfeld radiation condition [34] must be satisfied in order to ensure that all acoustic waves propagate freely towards infinity and that no reflections occur,

$$
\lim _{|\mathbf{r}| \rightarrow \infty}\left(\sqrt{|\mathbf{r}|}\left(\frac{\partial p(\mathbf{r})}{\partial|\mathbf{r}|}+j k p(\mathbf{r})\right)\right)=0 .
$$

The Helmholtz equation together with the associated boundary conditions, define a unique pressure field $p(\mathbf{r})$. Once this pressure field is calculated, derived acoustic quantities such as the acoustic velocity, the intensity and the acoustic power can be obtained.

\section{The WBM modelling procedure}

The general modelling procedure of the WBM to solve a generalised Helmholtz problem consists of the following four steps:

A. Partitioning of the considered problem domain into convex subdomains, 
B. Selection of a suitable set of wave functions for each subdomain,

C. Construction of the WB system matrices,

D. Solution of the system of equations, yielding the wave function contribution factors, and postprocessing of the dynamic variables.

These steps are first explained for a general Helmholtz problem and are then, as an illustration, applied to a 2D acoustic case.

\subsection{Partitioning of the problem domain}

When applied to bounded problems, the convexity of the considered domain is a sufficient condition for the WB approximations to converge towards the exact solution of the problem under study [17]. When the considered problem domain is non-convex, it is, in a first step, partitioned into a number of convex subdomains. When applied to unbounded problems, an initial partitioning of the unbounded domain into a bounded and an unbounded region by a truncation curve $\Gamma_{t}$ precedes the partitioning into convex subdomains [18], as illustrated by Figure 2 . The unbounded region exterior to $\Gamma_{t}$ is considered as one unbounded subdomain.

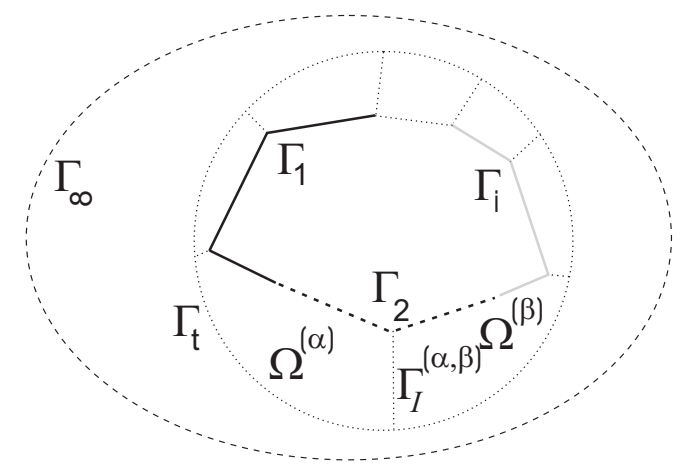

Figure 2: WB partitioning of an unbounded problem. Subdomain interfaces are shown in dotted lines $(\cdots)$.

The total problem domain $\Omega$ is subdivided into $N_{\Omega}$ number of non-overlapping subdomains $\Omega^{(\alpha)}\left(\alpha=1, \ldots, N_{\Omega}\right)$, which may be bounded or unbounded. On the interface $\Gamma_{I}^{(\alpha, \beta)}$ between two subdomains $\Omega^{(\alpha)}$ and $\Omega^{(\beta)}$, created in the partitioning procedure, continuity conditions need to be imposed

$$
\mathcal{B}_{I, l}^{(\alpha, \beta)}\left(\mathbf{u}^{(\alpha)}(\mathbf{r}), \mathbf{u}^{(\beta)}(\mathbf{r})\right)=0, \quad \mathbf{r} \in \Gamma_{I}^{(\alpha, \beta)} \quad l=1, \ldots, N_{H},
$$

with $\mathbf{u}^{(\alpha)}(\mathbf{r})$ and $\mathbf{u}^{(\beta)}(\mathbf{r})$ the dynamic field variables in the two adjacent subdomains and $\mathcal{B}_{I, l}^{(\alpha, \beta)}(\bullet, \star)=\mathcal{B}_{I, l}^{(\alpha)}(\bullet)+\mathcal{B}_{I, l}^{(\beta)}(\star)$ a general boundary differential operator expressing the continuity constraints on the fields $\bullet$ and $\star$ and their derived quantities. In order to obtain a well-posed system, one continuity condition is imposed for each of the $N_{H}$ dynamic variables on each subdomain.

For a 2D acoustic problem, two different approaches can be used: (i) directly enforce pressure continuity on one subdomain and normal velocity continuity on the other subdomain or (ii) apply impedance continuity conditions. Pluymers [35] has shown that the impedance coupling approach introduces artificial damping into the numerical model and has a beneficial effect on the WBM's efficiency. The impedance coupling conditions are given by

$$
\mathbf{r} \in \Gamma_{I}:\left(\mathcal{L}_{v}^{(\alpha)}\left(p^{(\alpha)}(\mathbf{r})\right)-\frac{p^{(\alpha)}(\mathbf{r})}{\bar{Z}_{\text {int }}}\right)=-\left(\mathcal{L}_{v}^{(\beta)}\left(p^{(\beta)}(\mathbf{r})\right)+\frac{p^{(\beta)}(\mathbf{r})}{\bar{Z}_{\text {int }}}\right)
$$

with $n^{(\alpha)}$ the local normal on the subdomain interface $\Gamma_{I}$, outwards of domain $\Omega^{(\alpha)}$, and $\bar{Z}_{\text {int }}$ an impedance coupling factor, typically chosen as $\rho c$ [35]. This continuity condition is enforced on each subdomain, for each interface, yielding two continuity conditions over each subdomain interface. 


\subsection{Field variable expansion}

The steady-state dynamic field(s) $u_{m}^{(\alpha)}(\mathbf{r})$ in each of the problem subdomains $\Omega^{(\alpha)}, \alpha=1, \ldots, N_{\Omega}$, are approximated by a solution expansion $\hat{u}_{m}^{(\alpha)}(\mathbf{r})$ in terms of $n_{m}^{(\alpha)}$ wave functions $\Phi_{w_{m}}^{(\alpha)}$

$$
u_{m}^{(\alpha)}(\mathbf{r}) \simeq \hat{u}_{m}^{(\alpha)}(\mathbf{r})=\sum_{w_{m}=1}^{n_{m}^{(\alpha)}} u_{w_{m}}^{(\alpha)} \Phi_{w_{m}}^{(\alpha)}(\mathbf{r})+\hat{u}_{p, m}^{(\alpha)}(\mathbf{r})=\boldsymbol{\Phi}_{\mathbf{m}}^{(\alpha)}(\mathbf{r}) \mathbf{u}_{\mathbf{m}}^{(\alpha)}+\hat{u}_{p, m}^{(\alpha)}(\mathbf{r}) .
$$

The wave function contribution factors $u_{w_{m}}^{(\alpha)}$ belonging to each of the wave functions $\Phi_{w_{m}}^{(\alpha)}(\mathbf{r})$ are gathered in the vector of degrees of freedom $\mathbf{u}_{\mathbf{m}}{ }^{(\alpha)}$. The row vector $\boldsymbol{\Phi}_{\mathbf{m}}^{(\alpha)}$ collects the $n_{m}^{(\alpha)}$ wave functions $\Phi_{w_{m}}^{(\alpha)}$. The term $\hat{u}_{p, m}^{(\alpha)}$ represents a particular solution resulting from the combined source terms $\mathcal{F}_{m}(\mathbf{r})$ in the right hand side of the inhomogeneous Helmholtz equation (1).

In accordance with the Trefftz principle [13], each of the wave functions $\Phi_{w_{m}}^{(\alpha)}(\mathbf{r})$ exactly satisfies the homogeneous part of the associated $m^{\text {th }}$ Helmholtz equation (1). In case of an unbounded subdomain, the wave functions are selected to additionally inherently fulfill the non-reflecting boundary condition (3) at $\Gamma_{\infty}$. Depending on the studied problem, these particular solutions usually take the form of dynamic fields generated by sources or forces in a homogeneous medium which extends to infinity. Consequently, irrespective of the values of the wave function contribution factors, the expansion (12) always satisfies the governing Helmholtz equation(s) (1).

Desmet [17] has proposed to select wave number components based on the characteristic dimensions of the problem at hand. For instance, for a bounded dynamic problem, the smallest rectangle (or rectangular box) circumscribing the considered 2D (3D) problem domains is used to determine the wave number components of the wave functions. The applied sets of wave functions within the WBM for the solution expansions for 2D and 3D bounded and unbounded subdomains and for various dynamic equations, can be found in the theoretical sections of the papers cited in Sections 5 and 7.

As an example, the wave function selection for a $2 \mathrm{D}$ unbounded acoustic problem is discussed here. In that case, the steady-state acoustic pressure field $p^{(\alpha)}(\mathbf{r})$ in an acoustic subdomain $\Omega^{(\alpha)}$ is approximated by a solution expansion $\hat{p}^{(\alpha)}(\mathbf{r})$

$$
p^{(\alpha)}(\mathbf{r}) \simeq \hat{p}^{(\alpha)}(\mathbf{r})=\sum_{w=1}^{n_{w}^{(\alpha)}} p_{w}^{(\alpha)} \Phi_{w}^{(\alpha)}(\mathbf{r})+\hat{p}_{q}(\mathbf{r})
$$

The wave function contributions $p_{w}^{(\alpha)}$ are the weighting factors for each of the selected wave functions $\Phi_{w}^{(\alpha)}$. Together they form the vector of degrees of freedom $\mathbf{p}^{(\alpha)}$. The corresponding a priori defined wave functions are collected in the row vector $\boldsymbol{\Phi}^{(\alpha)}$. The term $\hat{p}_{q}(\mathbf{r})$ represents a particular solution resulting from acoustic source terms $q$ in the right hand side of the inhomogeneous Helmholtz equation (4). The set of all $n_{W}=\sum_{\alpha=1}^{N_{\Omega}} n_{w}^{(\alpha)}$ acoustic wave function contributions $p_{w}^{(\alpha)}$ is collected in the column vector $\mathbf{p}$, while the row vector $\boldsymbol{\Phi}$ contains all $n_{W}$ wave functions. Depending on whether the subdomain is bounded or unbounded, different wave functions are selected.

For 2D bounded subdomains, two types of wave functions are distinguished, the so-called $\mathrm{r}$ - and s-set

$$
\sum_{w=1}^{n_{w}^{(\alpha)}} p_{w}^{(\alpha)} \Phi_{w}^{(\alpha)}(\mathbf{r})=\sum_{w_{r}=1}^{n_{w r}^{(\alpha)}} p_{w_{r}}^{(\alpha)} \Phi_{w_{r}}^{(\alpha)}(\mathbf{r})+\sum_{w_{s}=1}^{n_{w s}^{(\alpha)}} p_{w_{s}}^{(\alpha)} \Phi_{w_{s}}^{(\alpha)}(\mathbf{r})
$$

with the total number of wave functions in subdomain $\Omega^{(\alpha)}, n_{w}^{(\alpha)}$, defined as

$$
n_{w}^{(\alpha)}:=n_{w_{r}}^{(\alpha)}+n_{w_{s}}^{(\alpha)}
$$

The wave functions are defined as

$$
\Phi_{w}^{(\alpha)}(\mathbf{r})=\left\{\begin{array}{l}
\Phi_{w_{r}}^{(\alpha)}(x, y)=\cos \left(k_{x w_{r}}^{(\alpha)} x\right) e^{-j k_{y w_{r} y}^{(\alpha)} y} \\
\Phi_{w_{s}}^{(\alpha)}(x, y)=e^{-j k_{x w_{s}}^{(\alpha)} x} \cos \left(k_{y w_{s}}^{(\alpha)} y\right)
\end{array} .\right.
$$


The only requirement for the wave functions (16) to be exact solutions of the homogeneous part of the Helmholtz equation (4) is that the wave number components satisfy the associated dispersion relation

$$
\left(k_{x w_{r}}^{(\alpha)}\right)^{2}+\left(k_{y w_{r}}^{(\alpha)}\right)^{2}=\left(k_{x w_{s}}^{(\alpha)}\right)^{2}+\left(k_{y w_{s}}^{(\alpha)}\right)^{2}=k^{2} .
$$

As a result, an infinite number of wave functions (16) can be defined for expansion (13). Desmet [17] proposes to select the following wave number components

$$
\begin{aligned}
& \left(k_{x w_{r}}^{(\alpha)}, k_{y w_{r}}^{(\alpha)}\right)=\left(\frac{w_{1}^{(\alpha)} \pi}{L_{x}^{(\alpha)}}, \pm \sqrt{k^{2}-\left(k_{x w_{r}}^{(\alpha)}\right)^{2}}\right), \\
& \left(k_{x w_{s}}^{(\alpha)}, k_{y w_{s}}^{(\alpha)}\right)=\left( \pm \sqrt{k^{2}-\left(k_{y w_{s}}^{(\alpha)}\right)^{2}}, \frac{w_{2}^{(\alpha)} \pi}{L_{y}^{(\alpha)}}\right),
\end{aligned}
$$

with $w_{1}^{(\alpha)}=0,1,2, \ldots, w_{1, \max }^{(\alpha)}$ and $w_{2}^{(\alpha)}=0,1,2, \ldots, w_{2, \max }^{(\alpha)}$. The dimensions $L_{x}^{(\alpha)}$ and $L_{y}^{(\alpha)}$ represent the dimensions of the (smallest) bounding rectangle, circumscribing the considered subdomain $\Omega^{(\alpha)}$ as illustrated in Figure 3.

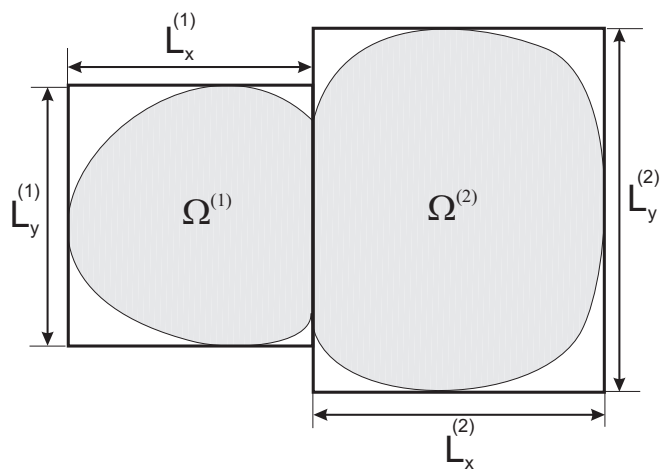

Figure 3: Illustration of the circumscribing bounding box dimensions.

Using this selection for the wave number components, the wave functions (16) represent a standing wave along one coordinate direction of the bounding box, multiplied with a propagating or evanescent component along the other direction. The particular solution $\hat{p}_{q}(\mathbf{r})$ in equation (13) represents the free field pressure field due to an acoustic point source at position $\left(x_{q}, y_{q}\right)$

$$
\hat{p}_{q}(x, y)=\frac{\rho \omega q}{4} H_{0}^{(2)}\left(k r_{q}\right),
$$

with $r_{q}=\sqrt{\left(x-x_{q}\right)^{2}+\left(y-y_{q}\right)^{2}}$ and $H_{0}^{(2)}(\bullet)$ the zero-order Hankel function of the second kind. The number $n_{w}^{(\alpha)}$ of wave functions for each subdomain $\Omega^{(\alpha)}$ is determined from the user-defined truncation factor $N$ by

$$
\begin{aligned}
w_{1, \text { max }}^{(\alpha)} & :=\max \left\{w_{1}^{(\alpha)} \in \mathbb{N} \mid \frac{w_{1}^{(\alpha)} \pi}{L_{x}^{(\alpha)}}<N k\right\}, \\
w_{2, \text { max }}^{(\alpha)}: & =\max \left\{w_{2}^{(\alpha)} \in \mathbb{N} \mid \frac{w_{2}^{(\alpha)} \pi}{L_{y}^{(\alpha)}}<N k\right\}, \\
& n_{w_{r}}^{(\alpha)}:=2\left(w_{1, \max }^{(\alpha)}+1\right), \\
& n_{w_{s}}^{(\alpha)}:=2\left(w_{2, \text { max }}^{(\alpha)}+1\right),
\end{aligned}
$$

and (15). The adopted truncation rule is frequency dependent and selects the number of wave functions such that the highest oscillatory wave number component of the $r$ - and the s-set is approximately equal to a user-defined truncation factor $N$ times the physical wavenumber $k$ of the considered problem. This truncation rule (20) is applied to all bounded subdomains such that the approximation field in adjacent subdomains exhibits a similar spatial variation along the interface. This way, a homogeneous approximation resolution is obtained throughout the problem domain. The number of wave functions increases approximately linearly with frequency. Numerical experiments have shown 
that for typical $2 \mathrm{D}$ acoustic applications a choice of $N=1, \ldots, 6$ gives a good balance between the computational cost of the WBM models and their prediction accuracy.

The wave functions for the unbounded domains are chosen to implicitly comply with not only the Helmholtz equation, but also with the Sommerfeld radiation condition (9) at $\Gamma_{\infty}$. This removes the need to explicitly impose a radiation condition, similarly as in the BEM. Herrera [36] shows that the following expansion $\hat{p}_{e}$, exterior to a circular truncation curve with radius $R_{t}$, yields a convergent set for the pressure field $p_{e}$, defined by a Neumann condition on the infinitely long cylinder with radius $R_{t}$ :

$$
\begin{aligned}
p_{e}(r, \theta) \simeq \hat{p}_{e}(r, \theta) & =p_{e, c 0} H_{0}^{(2)}(k r) \\
& +\sum_{n=1}^{n_{u}}\left(p_{e, c n} H_{n}^{(2)}(k r) \cos (n \theta)+p_{e, s n} H_{n}^{(2)}(k r) \sin (n \theta)\right),
\end{aligned}
$$

with $r$ and $\theta$ polar coordinates and $H_{n}^{(2)}(\bullet)$ the $n$th order Hankel function of the second kind. The contributions $p_{e, c 0}$, $p_{e, c n}$ and $p_{e, s n}$ are determined by the velocity boundary condition. From this expansion, the following wave function set for unbounded domains exterior to a circular truncation curve with radius $R_{t}$ is derived [20]:

$$
\Phi_{w}^{(u b)}(r, \theta)=\left\{\begin{array}{l}
\Phi_{w_{c}}^{(u b)}(r, \theta)=H_{w_{c}}^{(2)}(k r) \cos \left(w_{c} \theta\right) \\
\Phi_{w_{s}}^{(u b)}(r, \theta)=H_{w_{s}}^{(2)}(k r) \sin \left(w_{s} \theta\right)
\end{array},\right.
$$

with $w_{c}=0,1,2, \ldots, w_{c, \max }$ and $w_{s}=1,2,3, \ldots, w_{s, \max }$. As for bounded domains, the series of functions (23) is truncated to $n_{w}^{(u b)}$ functions, in order to be used in a numerical scheme. A truncation rule similar to equation (20) for the bounded domains determines the highest orders $w_{c, \max }$ and $w_{s, \max }$ of the Hankel functions used in the exterior wave function expansion [20]:

This truncation yields a total of

$$
\begin{aligned}
& w_{c, \text { max }}:=\max \left\{w_{c} \in \mathbb{N} \mid \frac{w_{c}}{2 R_{t}}<N k\right\}, \\
& w_{s, \text { max }}:=\max \left\{w_{s} \in \mathbb{N} \mid \frac{w_{s}}{2 R_{t}}<N k\right\} .
\end{aligned}
$$

$$
n_{w}^{(u b)}:=w_{c, \max }+w_{s, \max }+1
$$

wave functions in the unbounded domain. The physical interpretation of this rule is that a desired circumferential resolution of $\lambda /(2 N)$ is approximately obtained on the truncation $\Gamma_{t}$, with $\lambda=2 \pi / k$ the physical wavelength. Using this rule-of-thumb ensures a similar resolution of the functions in the unbounded and bounded subdomains. The functions (23) represent a harmonic distribution of the amplitude on the truncation, multiplied with the Hankel function $H_{n}^{(2)}(k r)$, describing the radial decay of an outgoing acoustic wave. The particular solution for a point source (19) can also be applied for unbounded domains. For unbounded problems another commonly used excitation for scattering problems is a plane wave source [18].

\subsection{Construction of the system of equations}

Within each subdomain $\Omega^{(\alpha)}$, the proposed solution expansion (12) always exactly satisfies the Helmholtz equation(s) (1), irrespective of the values of the unknown contribution factors $u_{w_{j}}^{(\alpha)}$. In case an unbounded subdomain is considered, the wave functions also fulfill the radiation condition (3) at $\Gamma_{\infty}$. However, the resulting dynamic field(s), such as for instance displacements, stresses etc, which can be obtained by applying an appropriate differential operator to the dynamic field variables $\mathbf{u}(\mathbf{r})$, may violate the imposed boundary conditions on the finite part of the boundary $\Gamma_{b}$ and interface conditions. The matrix system of equations is constructed by minimising these errors by applying a weighted residual approach. The residuals on the boundaries and interfaces of subdomain $\Omega^{(\alpha)}$ are orthogonalised with respect to a set of weighting functions $\tilde{\mathbf{t}}_{\bullet}^{(\alpha)}$

$$
\begin{array}{r}
\sum_{\beta=1, \beta \neq \alpha}^{N_{\Omega}} \sum_{l}^{N_{H}} \int_{\Gamma_{I}^{(\alpha, \beta)}} \tilde{\mathbf{t}}_{I, l}^{(\alpha)}(\mathbf{r}) \mathcal{B}_{I, l}^{(\alpha, \beta)}\left(\hat{\mathbf{u}}^{(\alpha)}(\mathbf{r}), \hat{\mathbf{u}}^{(\beta)}(\mathbf{r})\right) \mathrm{d} \Gamma \\
+\sum_{i} \sum_{l}^{N_{H}} \int_{\Gamma_{i}^{(\alpha)}} \tilde{\mathbf{t}}_{i, l}^{(\alpha)}(\mathbf{r})\left[\mathcal{B}_{i, l}\left(\hat{\mathbf{u}}^{(\alpha)}(\mathbf{r})\right)-\overline{\mathcal{B}}_{i, l}(\mathbf{r})\right] \mathrm{d} \Gamma=0 .
\end{array}
$$


The first line comprises the continuity conditions between two subdomains $\Omega^{(\alpha)}$ and $\Omega^{(\beta)}$ and the second line expresses the imposed boundary conditions on subdomain $\Omega^{(\alpha)}$. In general, the weighting function $\tilde{\mathbf{t}}_{\mathbf{0}}^{(\alpha)}(\mathbf{r})$ for each type of boundary and continuity condition can be derived from the underlying weighting functions $t_{m}^{(\alpha)}(\mathbf{r})$ with the same physical meaning as the field variables $u_{m}^{(\alpha)}$

$$
\tilde{\mathbf{t}}_{\bullet}^{(\alpha)}(\mathbf{r})=\mathcal{D}_{\bullet}(\mathbf{t}(\mathbf{r})),
$$

with $\mathbf{t}(\mathbf{r})$ the vector containing the weighting functions for the different components $t_{m}^{(\alpha)}$, and $\mathcal{D}_{\bullet}(\star)$ a problem and boundary condition dependent specific operator, generally determined based on a variational analysis of the considered problem [37]. Similarly to the Galerkin weighted procedure as often applied in the FEM, the weighting functions $t_{m}^{(\alpha)}(\mathbf{r})$ are expanded in terms of the same wave functions as for the field variable $u_{m}^{(\alpha)}(\mathbf{r})$

$$
t_{m}^{(\alpha)}(\mathbf{r})=\sum_{w_{m}=1}^{n_{m}^{(\alpha)}} \tilde{t}_{w_{m}}^{(\alpha)} \Phi_{w_{m}}^{(\alpha)}(\mathbf{r})
$$

Substitution of the field variable expansion (12) and the trial function expansion (28) yields an algebraic equation linking the unknown contribution factors of each wave function of subdomain $\Omega^{(\alpha)}$ to the unknown contribution factors of adjacent subdomains. For each subdomain $\Omega^{(\alpha)}$, a similar algebraic equation can be constructed. The enforcement that these $N_{\Omega}$ equations should hold for any combination of the weighting functions, results in a matrix system of equations of the following shape:

$$
[\mathbf{A}]\{\mathbf{u}\}=\mathbf{b},
$$

with $\mathbf{u}$ the vector containing all $n_{w}=\sum_{\alpha=1}^{N_{\Omega}} \sum_{m=1}^{N_{H}} n_{m}^{(\alpha)}$ unknown wave function contribution factors, $\mathbf{A}$ the system matrix and $\mathbf{b}$ the right-hand side vector, resulting from non-zero boundary conditions $\mathcal{B}_{i, l}(\mathbf{r})$ and particular solution terms $\hat{u}_{p, j}^{(\alpha)}(\mathbf{r})$.

For a 2D interior acoustic problem, the proposed expansion functions (16) exactly satisfy the Helmholtz equation (4) inside the domain and for a 2D exterior problem domain the expansion functions (23) fulfill in addition the Sommerfeld radiation condition (9). The boundary conditions (5)-(7) and subdomain continuity (11) are enforced through a weighted residual formulation.

For each subdomain, the residuals are orthogonalised with respect to a weighting function $\tilde{p}^{(\alpha)}(\mathbf{r})$ or its derivative. The weighted residual formulation, applying the introduced residual functions, is expressed as

$$
\begin{aligned}
& \int_{\Gamma_{v}^{(\alpha)}} \tilde{p}^{(\alpha)}(\mathbf{r}) R_{v}^{(\alpha)}(\mathbf{r}) \mathrm{d} \Gamma+\int_{\Gamma_{Z}^{(\alpha)}} \tilde{p}^{(\alpha)}(\mathbf{r}) R_{Z}^{(\alpha)}(\mathbf{r}) \mathrm{d} \Gamma \\
& -\int_{\Gamma_{p}^{(\alpha)}} \mathcal{L}_{v}^{(\alpha)}\left(\tilde{p}^{(\alpha)}(\mathbf{r})\right) R_{p}^{(\alpha)}(\mathbf{r}) d \Gamma+\sum_{\beta=1, \beta \neq \alpha}^{N_{\Omega}} \int_{\Gamma_{I}^{(\alpha, \beta)}} \tilde{p}^{(\alpha)}(\mathbf{r}) R_{I}^{(\alpha, \beta)}(\mathbf{r}) \mathrm{d} \Gamma=0 .
\end{aligned}
$$

The weighting functions $\tilde{p}^{(\alpha)}$ are expanded in terms of the same set of acoustic wave functions used in the pressure expansions (13)

$$
\tilde{p}^{(\alpha)}(\mathbf{r})=\sum_{w=1}^{n_{w}^{(\alpha)}} \tilde{p}_{w}^{(\alpha)} \Phi_{w}^{(\alpha)}(\mathbf{r})=\boldsymbol{\Phi}^{(\alpha)}(\mathbf{r}) \tilde{\mathbf{p}}^{(\alpha)} .
$$

Substitution of the pressure expansions (13) and the weighting function expansion (31) for subdomain $\Omega^{(\alpha)}$ into the weighted residual formulation (30) yields a set of $n_{w}^{(\alpha)}$ linear equations in the $n_{W}$ unknown wave function contribution factors. One such matrix equation is obtained for each subdomain. Combination of the equations of the $N_{\Omega}$ subdomains yields the acoustic WB model, consisting of $n_{W}$ algebraic equations in the $n_{W}$ unknown wave function contribution factors

$$
\left[\boldsymbol{A}_{a \boldsymbol{a}}\right]\{\mathbf{p}\}=\{\mathbf{b}\}
$$




\subsection{Solution and postprocessing}

In a final step, the system matrix of equation (29) is solved for the $n_{w}$ number of unknown wave function contribution factors. The backsubstitution of these values in the field variable expansions (12) leads to an analytical expression of the approximation of the field variables $\hat{u}_{m}^{(\alpha)}(\mathbf{r})$ in each of the subdomains $\Omega^{(\alpha)}$. Derivative quantities, such as acoustic velocities and structural displacements and stresses can be easily obtained by applying differential operators to the wave function sets. Since also evanescent components are used in the wave function sets, also near field effects can be captured.

\section{WBM Properties}

The WBM belongs to the family of deterministic approaches together with the FEM and the BEM. Nevertheless, due to the fundamentally different choice of approximation functions and domain/boundary discretisations, a different modelling procedure and different properties are obtained. This section briefly compares the WB modelling approach with the FEM and the BEM and highlights the advantages and disadvantages. It should be noted that all the methods are considered in their conventional forms, without recent developments and enhancements. The main motivation here is to give a comparison of the working principles of the methods.

Problem discretisation and degrees of freedom. The FEM divides the problem domain into a large number of small elements. The DOFs in an FE model are the nodal values of the field variables, and inside the elements the dynamic field is approximated using simple polynomial shape functions. The BEM discretises the boundaries of the problem domain instead of the domain itself. Depending on the formulation (direct or indirect), the DOFs can be the nodal values of the field variables or the potentials. Compared to the FEM, the number of elements is less, however the system is still composed of large number of small elements. As frequency increases and wavelengths shorten, the $\mathrm{FE}$ and $\mathrm{BE}$ meshes need to be refined to retain a similar accuracy as driven by interpolation and pollution errors $[3,4]$. Contrarily, the WBM partitions the domain into a small number of large subdomains, which are frequency independent. The only prerequisite is that the bounded subdomains have to be convex [17]. The general strategy in the partitioning process is to divide the domain in to the lowest possible number of convex subdomains, while avoiding steep changes in boundary conditions within a subdomain. The selected wave functions are frequency dependent, and they are exact solutions of the governing equations. The DOFs are the contribution factors of each of the wave functions and do not have a direct physical meaning. To obtain a finer spatial resolution of the dynamic field, the number of wave functions is increased, without changing the domain decomposition. Refining the model consequently does not require a remeshing of the domain.

Problem geometrical complexity. Due to the fine discretisation, the FEM and the BEM have almost no restrictions regarding the geometrical complexity. For the WBM on the other hand, all subdomains need to fulfil the convexity requirement. Non-convex domains have to be partitioned into an as small as possible number of large, convex subdomains. As the number of subdomains increases, so does the number of interfaces and consequently the integration length, leading to an increase in computational load. As explained later, the main computational cost in WB models results from the construction of the system of equations as it involves the integration of products of highly oscillating wave functions. As the system matrix is ill conditioned, these integrations should be performed accurately. Taking these restrictions into account, the smallest number of convex subdomains is preferred, minimising the total integration length. Consequently, the WBM shows its full efficiency for moderately complex geometries.

To increase the applicability of the method, two recent developments partially relax those constraints: the multilevel framework [38, 39] and hybrid approaches [37, 40, 41]. The multi-level framework alleviates the problem of multiple scatterers or inclusions. For such problems, the partitioning into convex domains could lead to a very large number of subdomains if possible at all. The Multi-Level WBM allows modelling of the scatterers or the inclusions on different levels, as if they are alone and avoids the complex domain division process. The system is then built up by combining all levels using the superposition theory while keeping the efficieny of the WBM.

Hybrid approaches combine the best properties of two prediction techniques: (i) the ability of element-based techniques to tackle the geometrically complex parts of the problem at hand, and (ii) the WBM to deal in a more efficient way with the large, convex parts of the problem domain. The multi-level and hybrid approaches are discussed in more detail in Section 5. 
System matrix properties. In general, the system matrices of the FEM are real-valued, large, frequency independent and sparsely populated with a banded structure. These properties allow for an efficient solution, and a reuse of the matrices for different frequencies. Nevertheless, for some problems, like poroelastic materials, the material properties are complex and frequency dependent. In this case, the FE matrices, which are complex, have to be recalculated for each frequency, hampering the efficient solution and also the applicability of modal reduction schemes. In contrast to FE matrices, the BE and WB matrices are always complex, frequency dependent and fully populated. The BE matrices are smaller compared to the FE matrices and the WB ones are even smaller than the BE's. On the other hand, both for the BEM and the WBM, the matrices need to be reconstructed for every frequency of interest. As is common for Trefftz approaches, also the WBM yields ill-conditioned matrices [29, 42]. However, Desmet [17] has shown that, despite this ill-conditioning, an accurate solution can be obtained by applying direct solution methods if the WB matrices satisfy both Picard conditions [43, 44].

Accuracy of the derived variables. Since the FEM commonly applies polynomial shape functions to approximate the primary response variables, the higher order derived quantities are approximated less accurately. Although the BEM also uses polynomial shape functions for the primary response variables, the field values are calculated through Green's function. The derived variables can be calculated by analytical derivation without losing accuracy. When it comes to the WBM, since derivatives of wave functions are again wave functions, with the same spatial resolution, derived variables are predicted with the same spatial resolution as the primary variables.

Construction of the system matrices. Building the WB models involves the evaluation of integrals of highly oscillatory functions and building the BE models requires computation of singular integrals. They both need extra care as compared to the construction of the FE matrices, which only requires the integration of simple polynomial functions. For the BEM, coordinate transformations or special integration rules are used to avoid the singularities. For the nonsingular parts it is more straightforward, however, the computational load is still high because of the calculation of Green's function for all the possible interactions of the elements. In the end, although the system matrices are smaller than FEM's, constructing them is computationally more demanding. For the WBM, due to the ill-conditioning of the WB matrices, the integrations must be performed carefully to obtain a sufficient accuracy of the matrix coefficients. Numerical integration, by applying the Gauss-Legendre integration rule, was shown to be the most efficient for the kind of integrals to be solved for a WB scheme, since an efficient matrix multiplication can be applied [45]. This numerical integration technique is applied with a fixed number of quadrature points per smallest wavelength resulting from the selected wave number components in the wave function sets. Even so, the system building takes more time as compared to the FEM.

Solution of the system matrices. Although the FE matrices are in general sparse and symmetric, because of the large number of FE degrees of freedom, the solution of the FE matrices is the computationally most demanding part of its process. When compared with the solution of the BEM matrices, it is still faster because the reduction in the size of the matrix is not enough to overcome the difference between solving a fully populated and a sparse matrix. When it comes to the WBM and when problems of a moderate geometrical complexity are considered, this statement is not valid anymore because the reduction in the size of the matrix is substantially higher as compared to the BEM. Therefore the solution of the WBM matrices becomes the fastest among the three.

Overall computational performance. Considering all of the above statements, it can be concluded that all three methods have their advantages and disadvantages and defining a clear winner is not always possible. This is mainly due to the fact that the overall computational performance will mainly depend on the application. Therefore, without making bold statements, a general overview can be given as follows. The FEM and the BEM have advantages for highly complex geometries since that they do not have restrictions regarding the problem geometry. The WBM, on the other hand, shows its efficiency for moderately complex geometries and loses its edge when applied on highly complex ones. Being said that, the WBM is more efficient than the FEM and the BEM when used for the right application. Since it applies exact solutions of the governing partial differential equation(s), it has a better convergence rate which allows the method to tackle a higher frequency range than the element-based techniques. Besides the complexity of the problem, another important subdivision can be made concerning the type of the problem, i.e. if it is bounded or unbounded. As a general rule, the FEM is better suited for the bounded problems and the BEM for the unbounded, 
because the FEM needs special treatments to be able to solve the unbounded problems. This makes it lose its computational appeal against the BEM since the BE formulation inherently complies with the imposed outgoing field conditions at infinity. As for the WBM, the efficiency is not affected by the type of the problem as it can inherently solve the unbounded problems by applying unbounded wave functions. This makes the WBM an appealing alternative for both the FEM and the BEM for moderately complex geometries. It is because of this appeal that the method has been applied to various fields and extended to different forms, i.e. the hybrid and multi-level approaches, which are detailed in the following sections.

\section{WBM state of the art}

The concept of the WBM has been introduced by Desmet [17] and since then has been the topic of continuous research. This section gives a short overview of the problem types that can currently be tackled by the WBM, and discusses two different enhancements to overcome some of the WBM's limitations and to increase its applicability and versatility: the multi-level approach and hybrid approaches. Also recent application areas are described.

\subsection{Current capabilities of the basic version of the WBM}

As is already indicated in Section 3, every dynamic problem, of which the mathematical formulation is given by or can be cast into a (number of) Helmholtz equation(s), can be tackled by the WBM. So far, the WBM has been applied to the following problem types:

Interior acoustic problems. The WBM was originally developed for interior acoustic problems [17]. This work was further continued by van Hal [37], who focussed on 2D acoustic problems consisting of one single or a number of subdomains. Pluymers continued this research and enhanced subdomain coupling by applying mixed interface conditions [35]. This way, artificial damping was introduced into the numerical system, leading to a more stable convergence. Furthermore, the method was extended to 3D problems [46]. Recently, special purpose enrichment functions for 2D acoustic problems with corner singularities have been discussed [47].

Exterior acoustic problems. In order to deal with unbounded and coupled bounded-unbounded problem domains, Pluymers [35, 48] introduced the concept of the initial partitioning of the problem into a bounded and an unbounded subdomain, by defining the truncation boundary $\Gamma_{t}$. He defined 2D unbounded wave function sets, not only satisfying the homogeneous Helmholtz equation, but also complying with the Sommerfeld radiation condition at $\Gamma_{\infty}$ [34]. Bergen et al. extended this work to 3D unbounded acoustic problems [49]. In a next step, the formulation proposed for unbounded problems was extended to semi-unbounded problems, enabling the modelling of diffraction problems and for instance domains delimited by a rigid ground, in both 2D [50] and 3D [20].

Plate bending problems. The application of the WBM to dynamic plate bending problems was first studied by Desmet [17]. Later, Vanmaele et al. [51] studied this topic more profoundly. The presence of stress singularities, occurring in corner points of the plate domain was identified, and special purpose enrichment functions were included in the wave function set to incorporate this behaviour [52]. This way, an important gain in accuracy is obtained.

Plate membrane problems. Vanmaele et al. [53] also studied membrane problems by casting the Navier equations into two Helmholtz equations using the Helmholtz decomposition theorem [54]. Also for this type of structural dynamic problems, enrichment of the wave function set by special purpose enrichment functions improves the convergence rate when singularities are present in corner points of the domain.

Assemblies of flat shells. When two flat plates are connected to each other under a certain angle, the in and out of plane displacement components greatly influence each other. Vanmaele [55] combined the WB approximation fields for membrane and bending behaviour to model shell behaviour for assemblies consisting of several flat plates [56].

Coupled vibro-acoustic problems. The WB developments for bounded acoustic and structural models were combined to predict the coupled response of an interior vibro-acoustic problem [17,37]. This approach has also been applied to 2D scattering problems containing a structural component [18, 57]. 
Poroelastic material modelling. Desmet [17] touched upon this topic and indicated a possible way to decouple the dynamic Biot equations into three Helmholtz equations. Lanoye et al. [58] developed a WB procedure to study patchworks of porous materials. In that work, an equivalent fluid representation is applied; as such only one Helmholtz equation needs to be considered. Deckers et al. further developed the WBM for the Biot equations for Cartesian [59] and axisymmetric coordinate systems [60]. Also special purpose enrichment functions have been derived to counter the adverse effect of singularities [61].

\subsection{Multi-level WBM}

In the case an unbounded problem geometry contains several scatterers, the WBM loses it attractiveness. The truncation $\Gamma_{t}$ needs to enclose all scatterers and inside this truncation, the convexity requirement may lead to a very large number of subdomains. When, for instance, a number of circular scatterers are considered, it is even impossible to obtain convex subdomains. The same holds for a bounded subdomain with (a number of) inclusion(s). To overcome these difficulties, the concept of multi-level modelling was first introduced for scattering problems. The procedure is depicted in Figure 4 for a configuration with two scatterers $S_{1}$ and $S_{2}$. Each of the scatterers in the problem setting is considered as a different level. In every level, the scattering of just one particular object is taken into account. These different levels can be modelled using the existing unbounded WB methodology. The incident field for one level is the scattered field of the remaining objects and the incident field from external excitations. By applying the superposition principle and linking all levels together via a weighted residual approach, a numerical model is obtained. As such, dynamic problems containing a number of scatterers can be tackled in a much more efficient way.

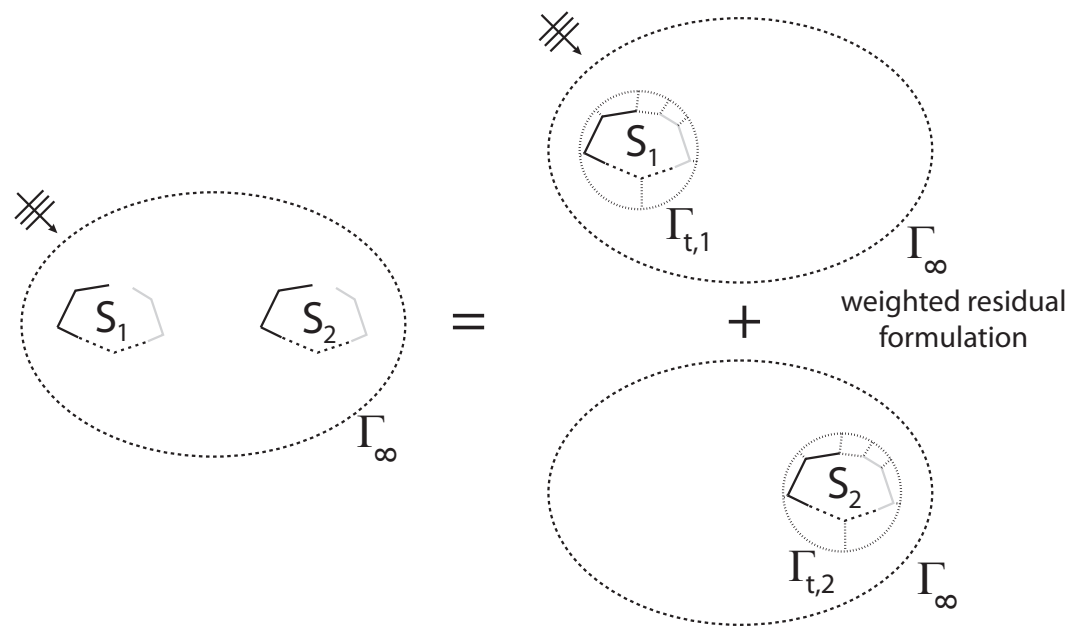

Figure 4: Concept of the multi-level approach [62].

The concept was first proposed by Van Genechten et al. [38] for 2D acoustic scattering problems. Later, Bergen [20] extended the concept to 3D acoustic scattering problems. The multi-level concept is, however, not restricted to unbounded problems. Bounded problems, containing a number of inclusions, can be tackled in a completely similar fashion. Each of the inclusions is studied in a different level, and the surrounding domain is taken into account in an additional, separate level. The 'bounded level' describes the dynamic fields in the bounded domain without inclusions. Van Genechten et al. [39] have applied the multi-level to bounded subdomains with inclusions to 2D acoustic applications and 2D plate membrane problems. Vergote [62] has extended the procedure to plate bending problems.

\subsection{Hybrid WB methods}

The second class of enhancements applies the combined use of two numerical methods to one problem under study. Each method is applied according to its own strengths. This way, a strong hybrid approach can be obtained, taking benefit of the best properties of two approaches. 
Hybrid Finite Element - Wave Based Method (FE-WBM). Whereas the WBM is extremely efficient for large problem domains with a simple geometry, the FEM has almost no restrictions regarding the geometrical complexity of a problem domain, although it suffers from dispersion and pollution errors. A hybrid FE-WBM is developed to combine the 'best of two worlds'. The main idea is explained in Figure 5. The WBM and the FEM are applied in nonoverlapping regions. Large, geometrically simple parts of the problem domain are modelled by the WBM whereas the FEM focusses on geometrically complex regions.

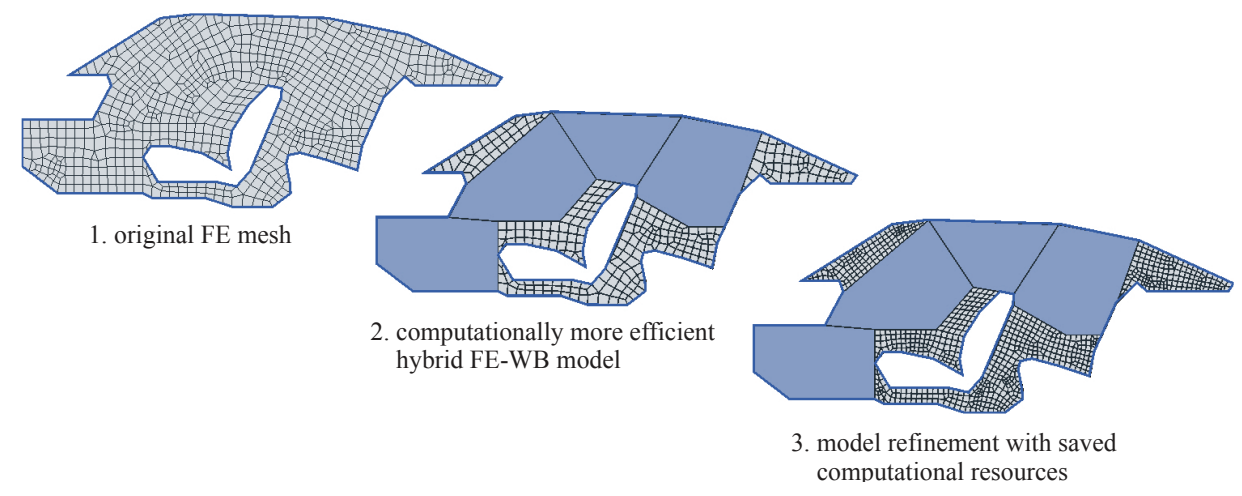

Figure 5: Concept of hybrid FE-WBM approach [37]

The hybrid FE-WBM approach was proposed by van Hal [37], who developed two ways of coupling the methods together on the interfaces: a direct and an indirect coupling approach. van Hal applied this procedure to 2D bounded acoustic problems [63, 64]. Pluymers [35] extended the approach towards 3D bounded acoustic problems and validated it for several problems of industrial complexity. Vanmaele [55] applied the hybrid methodology to the modelling of membrane problems and for combined plate-beam problems. Van Genechten et al. [40] developed a hybrid FE-WBM for vibro-acoustic problems, modelling the acoustic domain with the WBM and the structural part with the FEM. Van Genechten [19] also proposed to use a modal reduction in the FE subdomain, for a further speed up of the hybrid approach. Bergen [20] proposed a hybrid approach for 2D acoustics, where a finite domain is modelled by the FEM and coupled to an unbounded WB domain outside a circular truncation $\Gamma_{t}$. This approach can be seen as an efficient alternative to the classical Dirichlet-to-Neumann-map (DtN) [65]. Recently, a hybrid FE-WBM was proposed for poroelastic domains [66]. Finally, Jonckheere et al. [67] developed a hybrid approach to model vibro-acoustic problems with trim layers. The WBM is applied to the acoustic part of the problem whereas the FEM allows the modelling of the trim layer in great detail.

Hybrid Boundary Element - Wave Based Method (BE-WBM). Following the working principles of the multi-level approach and and the philosophy of the hybrid FE-WBM, recently, a hybrid BE-WBM has been developed, which can be deployed for unbounded acoustic problems, containing geometrically simple as well as complex scatterers. In such a setting, the multi-level WB approach still loses its efficiency, since a large number of subdomains are required in the levels containing the geometrically complex scatterers. Atak et al. [41] proposed the hybrid BE-WBM, where the geometrically simple levels, such as circles, are described by the WBM and the complex scatterers, which would require a large number of WB subdomains, are gathered in one level which is described by the BEM. With this novel approach, a broad spectrum of applications can be addressed. For instance the combination of simple and complex scatterers together with heterogeneities and open boundaries in unbounded acoustic domains can be tackled, which cannot be modelled by the WBM and the BEM in their pure forms.

Hybrid Wave Based Method - Statistical Energy Analysis (WBM-SEA). The WBM is a deterministic method, that aims to relieve the severe frequency limitations as imposed by element-based techniques. However, at high frequencies, the effect of variability on for instance material properties and problem geometry, which are inevitably present in a real system, have to be accounted for. The study of one deterministic model is no longer representative to predict the response for an ensemble of products. Typically space- and frequency-averaged responses are then calculated by applying a statistical approach such as the SEA [6]. However, when combining different problem types, especially in 
built-up structures, some parts of the problem may still behave deterministically, whereas other parts already behave statistically. As such, the cost of a full deterministic model cannot longer be justified, but also the SEA can no longer be applied on the full system. Shorter and Langley [68] proposed a hybrid FE-SEA to handle this type of mixed problems. Vergote [69] substituted the FE part in such an approach for the WBM, offering an increased efficiency, especially in the mid-frequency range.

\section{Recent application areas}

Vergote [62] improved the WBM for plate bending model by adding point connections (masses, dampers, springs and tuned vibration absorbers), allowing to simulate passive (and by extension also active) control treatments. He has shown that only an extra row and column have to be added to the WB matrix system of equation for every point connection, without changing the original system. As such, optimisation for the ideal position of a number of point connections becomes very efficient.

Vergote [62] also studied different ways to perturb WB models of plates to take into account variability in the mid frequency range. It has been shown that by applying randomly distributed point masses to a plate, a Monte Carlo simulation can be performed in a much more efficient way than by using the FEM.

Successively, the WBM has been used by D'Amico et al. to perform efficient evaluations of the frequency averaged input power into plates by using a Lorentzian function as a weighting function [70]. Due to the mathematical features of the Lorentzian, the frequency average procedure can be evaluated straightforwardly for causal systems without the need of the response computation at several frequencies.

Deckers et al. [45] have proposed the use of B-splines for the description of curved domain boundaries within the WBM. In this way, curved boundaries do not need to be divided into a large number of small segments, removing the restrictions on the integration imposed by segmentation. Many CAD-packages apply B-splines for geometry description, and the possibility to use this same geometrical description for the WB model allows for easier model construction, while retaining the geometrical features from the original design.

Koo [71] implemented the design sensitivity analysis of a weakly coupled structural-acoustic problem using the WBM. The adjoint variable method is used to compute the sensitivity in an efficient way. The adjoint variable is defined from a structural reanalysis using an adjoint load composed of the system matrix and an evaluation of the wave functions of each boundary. The optimal design, minimising the radiated noise from a vibrating structure, can then be obtained through the optimisation process.

\section{Outlook}

The research on the WBM is still actively progressing, to make the tool more attractive and applicable for a wide range of applications. Four ongoing/future focal points are highlighted here.

As indicated in the introduction section, effective and efficient vibration and acoustic modelling methods are required to design and analyse products with good noise and vibration performance. No single method allows for meaningful calculations over the whole frequency range of interest. Every developed tool has its own strengths and weaknesses and should be applied accordingly. The WBM shows its full efficiency for geometrically simple problems. To be used for problem cases of industrial complexity, it has to be combined in a hybrid fashion with other existing methods, such as FEM, BEM and SEA. In this sense it can be applied to overcome computational burdens of these methods. Further developments of hybrid combinations and their application to industrial applications is still one of the core focal points for the upcoming years.

A second main topic is the combination of efficient WB models and vibro-acoustic measurement setups. Since many setups have a relatively simple geometry, the WBM can provide a powerful tool to validate the outcome of experiments and the effect of loading and boundary conditions. An example can be found in [60]. The WBM recently also has been applied to validate experiments on the KU Leuven Sound Box. This is a lightweight box which allows to characterise the absorption and the insertion loss of lightweight panels, e.g. see [72]. In this aspect also optimisation strategies will be applied and developed.

The WBM allows easy model refinement as compared to for instance FEM, since no remeshing is required. In certain optimisation scenarios where only parts of the full model are optimised, the ML-WBM allows the reuse of the 
rest of the model. This intrinsic ability makes it possible to exploit the efficiency of the method further for optimisation problems. A first application case is on the design of acoustic lenses [73]. A challenging step will be applying shape optimisation to a WB model since in WB models, the dimensions of the model are used to define the wave number components of the wave functions.

A fourth track will focus on the addition of non-determinism in WB models to make higher frequency simulations more meaningful. In the mid frequency region the response of a dynamic system becomes increasingly sensitive to uncertainty and variability of the model parameters. The coupling of WBM and SEA [69] and study of Monte Carlo simulations using WB models [62] formed a first step. The next step will be the development of a fuzzy-WB method that can predict the range of the possible responses of a non-deterministic system. In this context, the application of parametric model reduction techniques to the WBM will also be studied to improve its efficiency.

\section{Conclusion}

This paper discusses the state of the art of the WBM, summarising fifteen years of continuous research. The WBM is based on an indirect Trefftz approach and is specifically developed to solve dynamic problems governed by a (number of) Helmholtz equations.

Firstly, the problem definition for a generalised Helmholtz problem is discussed. Next, the four-step modelling procedure of the WBM is elaborated. By partitioning the problem domain under study into a number of large convex subdomains, the convergence of the method is assured. When considering an unbounded domain, an initial partitioning of the problem domain into an unbounded subdomain and a bounded region by a truncation circle or sphere precedes the partitioning into convex subdomains. Coupling conditions have to be imposed on the primary and derived variables on the interfaces to ensure continuity between adjacent subdomains. Within each subdomain, the field variables, resulting from the governing Helmholtz equations, are approximated by a weighted set of wave functions. The wave functions are defined a priori, exactly fulfilling the governing dynamic equations. For an unbounded domain, the wave functions are selected such that they also comply with the non-reflecting boundary condition at infinity, representing a purely outgoing field. In a third step, the residuals on the boundaries and interfaces are minimised by applying a weighted residual approach, yielding a system of equations which can be solved for the unknown contribution factor of each wave function in a final step.

Also the properties of the WBM are elaborated and compared to those of FEM and the BEM. Although the WB matrices are ill-conditioned, they still yield accurate solutions. The WBM matrices are generally frequency dependent, complex and small, and high convergence rates are obtained. These properties enable the solution of WB models towards higher frequencies as compared to FE models. The main restrictions on the use of the WBM are imposed by the convexity requirement. As such, the WBM is mainly efficient to model problems with a limited geometrical complexity.

The current state of the art of the WBM is discussed. The studied problem types are summarised. Currently, the WBM can be applied for 2D and 3D interior and exterior acoustic problems, for plate membrane and plate bending problems, for plate assemblies, for vibro-acoustic problems and for $2 \mathrm{D}$ and $2.5 \mathrm{D}$ poroelastic materials. Also general enhancements to the Wave Based Method are discussed: the multi-level approach, which allows to efficiently model problems with multiple scatterers or inclusions, and the family of hybrid approaches (the WBM combined with FEM, BEM and SEA) which use each method according to its own strengths. Also recent applications are listed. The last section discusses the outlook for the WBM.

\section{Acknowledgments}

The research of Elke Deckers was partially funded by the Research fund KU Leuven; during the review process she was a postdoctoral fellow of the Fund for Scientific Research - Flanders (F.W.O). The institute for Promotion of Innovation by Science and Technology Flanders (Belgium) (IWT-Vlaanderen) is gratefully acknowledged for the support of the doctoral research of Laurens Coox, Hendrik Devriendt and Stijn Jonckheere. The authors also gratefully acknowledge the European Commission for their support through the ITN Marie Curie project GA-290050 GRESIMO and the EID Marie Curie project GA-316422 eLiQuiD. The IWT Flanders within the ASTRA and the HEV-NVH project, the Fund for Scientic Research - Flanders (F.W.O.), Belgium and the Research Fund KU Leuven are also gratefully acknowledged. 


\section{References}

[1] O. C. Zienkiewicz, R.L. Taylor, J.Z. Zhu, and P. Nithiarasu. The Finite Element Method - The three volume set. Butterworth-Heinemann, 6th edition, 2005

[2] O. Von Estorff. Boundary Elements in Acoustics: Advances and Applications. WITpress, 2000.

[3] P. Bouillard and R. Ihlenburg. Error estimation and adaptivity for the finite element method in acoustics: 2D and 3D applications. Computer Methods in Applied Mechanics and Engineering, 176:147-163, 1999.

[4] R. Freymann. Advanced numerical and experimental methods in the field of vehicle structural-acoustics. Hieronymus Buchreproduktions $\mathrm{GmbH}, 2000$.

[5] S. Marburg. Six boundary elements per wavelength: is that enough? Journal of Computational Acoustics, 10:25-51, 2002.

[6] R. Lyon and R. DeJong. Theory and application of statistical energy analysis. Butterworth-Heinemann, 2nd edition, 1995.

[7] Y.A. Erlangga. Advances in iterative methods and preconditioners for the Helmholtz equation. Archives of Computational Methods in Engineering, 15:37-66, 2008.

[8] R.R. Craig Jr. and A.J. Kurdila. Fundamentals of Structural Dynamics. John Wiley \& Sons, New York Chichester Brisbane Toronto Singapore, 2nd edition, 2005.

[9] T. Sakuma and Y. Yasuda. Fast multipole boundary element method for large-scale steady-state sound field analysis. Part I: setup and validation. Acustica, 88:513-525, 2002.

[10] Y. Yasuda and T. Sakuma. Fast multipole boundary element method for large-scale steady-state sound field analysis. Part II: examination of numerical items. Acustica, 89:28-38, 2002.

[11] C. Farhat, I. Harari, and L.P. Franca. The discontinuous enrichment method. Computer Methods in Applied Mechanics and Engineering, 190:6455-6479, 2001.

[12] J. Melenk and I. Babuška. The partition of unity finite element method: Basic theory and applications. Computer Methods in Applied Mechanics and Engineering, 139:289-314, 1996.

[13] E. Trefftz. Ein Gegenstück zum Ritzschen Verfahren. In Proceedings of the $2^{\text {nd }}$ International Congress on Applied Mechanics, Zurich, Switzerland, pages 131-137, 1926.

[14] B. Mace and P. Shorter. Energy flow models from finite element analysis. Journal of Sound and Vibration, 233:369-389, 2000.

[15] L. Maxit and J.-L. Guyader. Estimation of SEA coupling loss factors using a dual formulation and FEM modal information, part I: Theory. Journal of Sound and Vibration, 239:907-930, 2001.

[16] R.S. Langley and J.A. Cordioli. Hybrid deterministic-statistical analysis of vibro-acoustic systems with domain couplings on statistical components. Journal of Sound and Vibration, 321:893-912, 2009.

[17] W. Desmet. A wave based prediction technique for coupled vibro-acoustic analysis. KULeuven, division PMA, PhD. thesis 98D12, 1998.

[18] B. Pluymers, B. Van Hal, D. Vandepitte, and W. Desmet. Trefftz-based methods for time-harmonic acoustics. Archives of Computational Methods in Engineering, 14:343-381, 2007.

[19] B. Van Genechten. Trefftz-based mid-frequency analysis of geometrically complex vibro-acoustic problems - hybrid methodologies and multi-level modelling. KULeuven, division PMA, PhD. thesis 2010D08, 2010.

[20] B. Bergen. Wave based modelling techniques for unbounded acoustic problems. KULeuven, division PMA, PhD. thesis 2011D07, 2011.

[21] L. Franca and C. Farhat and A. Macedo and M. Lesoinne. Residual-free bubbles for Helmholtz equation. International Journal for Numerical Methods in Engineering, 40:4003-4009, 1997.

[22] R. Tezaur and L. Zhang and C. Farhat. A discontinuous enrichment method for capturing evanescent waves in multiscale fluid and fluid/solid problems. Computer Methods in Applied Mechanics and Engineering, 197:1680-1698, 2008.

[23] G. Gabard. Discontinuous Galerkin methods with plane waves for timeharmonic problems. Journal of Computational Physics, 225:1961$1984,2007$.

[24] T. Strouboulis and K. Copps and I. Babuška. The generalized finite element method. Computer Methods in Applied Mechanics and Engineering, 190:4081-4193, 2001.

[25] E. Perrey-Debain and J. Trevelyan and P. Bettess. Wave boundary elements: a theoretical overview presenting applications in scattering of short waves. Engineering Analysis with Boundary Elements, 28:131-141, 2004.

[26] G.H. Koopmann and L. Song and J.B. Fahnline. A method for computing acoustic fields based on the principle of wave superposition. Journal of the Acoustical Society of America, 86:2443-2438, 1989.

[27] M. Ochmann. The source simulation technique for acoustic radiation problems. Acustica, 81:512-527, 1995.

[28] M.A. Goldbert and C.S. Chen. The MFS for potential, Helmholtz and diffusion problems, Chapter 4, Boundary Integral Methods: Numerical and Mathematical Aspects. WIT Press and Computational Mechanics Publ. Boston, Southampton, 1999.

[29] T. Huttunen and P. Gamallo and R.J. Astley. Comparison of two wave element methods for the Helmholtz problem. Communications in Numerical Methods in Engineering, 25:35-52, 2009.

[30] Q.-H. Qin. Formulation of hybrid Trefftz finite element method for elastoplasticity. Applied Mathematical Modelling, 29:235-252, 2005.

[31] H. Riou, P. Ladèveze, and P. Rouch. Extension of the variational theory of complex rays to shells for medium-frequency vibrations. Journal of Sound and Vibration, 272:341-360, 2004

[32] L. Kovalevsky and P. Ladevèze and H. Riou. The Fourier version of the Variational Theory of Complex Rays for medium-frequency acoustics . Computer Methods in Applied Mechanics and Engineering , 225-228:142-153, 2012.

[33] A. Pierce. Acoustics: An Introduction to Its Physical Principles and Applications. McGraw-Hill series in mechanical engineering, McGrawHill, 1981.

[34] D. Colton and R. Kress. Inverse acoustic and electromagnetic scattering theory. Springer-Verlag, Berlin, Heidelbert, New York, 2nd edition, 1998.

[35] B. Pluymers. Wave based modelling methods for steady-state vibro-acoustics. K.U.Leuven, division PMA, PhD. thesis 2006D04, Leuven, 2006.

[36] I. Herrera. Boundary Methods: an Algebraic Theory. Applicable mathematic series. Pitman Advanced Publishing Programm, 1984. 
[37] B. van Hal. Automation and performance optimization of the wave based method for interior structural-acoustic problems. PhD Thesis, Faculty of Engineering, Katholieke Universiteit Leuven, 2004.

[38] B. Van Genechten, B. Bergen, D. Vandepitte, and W. Desmet. A Trefftz-based numerical modelling framework for Helmholtz problems with complex multiple scatterer configurations. Journal of Computational Physics, 229(18):6623-6643, 2010.

[39] B. Van Genechten, K. Vergote, D. Vandepitte, and W. Desmet. A multi-level wave based numerical modelling framework for the steady-state dynamic analysis of bounded Helmholtz problems with multiple inclusions. Computer Methods in Applied Mechanics and Engineering, 199:1881-1905, 2010.

[40] B. Van Genechten, D. Vandepitte, and W. Desmet. A direct hybrid finite element - Wave based modelling technique for efficient coupled vibro-acoustic analysis. Computer Methods in Applied Mechanics and Engineering, 200:742-764, 2011.

[41] O. Atak and B. Bergen and D. Huybrechs and B. Pluymers and W. Desmet. Coupling of Boundary Element and Wave Based Methods for efficient solution of complex multiple scattering problems. Journal of Computational Physics (in press), http://dx.doi.org/10.1016/j.jcp.2013.10.034, 2013.

[42] A. P. Zieliński and I. Herrera. Trefftz method: fitting boundary conditions. International Journal on Numerical Methods in Engineering, 24:871-891, 1987.

[43] J.M. Varah. A practical examination of some numerical methods for linear discrete ill-posed problems. SIAM Review, 21:100-111, 1979.

[44] J.M. Varah. Pitfalls in the numerical solution of linear ill-posed problems. SIAM Journal on Scientific and Statistical Computing, 4:164-176, 1983.

[45] E. Deckers, B. Drofmans, B. Van Genechten, B. Bergen, D. Vandepitte, and W. Desmet. Spline-based boundaries: a first step towards generic geometric domain descriptions for efficient mid-frequency acoustic analysis using the Wave Based Method. Journal of Computational and Applied Mathematics, 235:2679-2693, 2011.

[46] B. Van Genechten, O. Atak, B. Bergen, E. Deckers, S. Jonckheere, J.S. Lee, A. Maressa, K. Vergote, B. Pluymers, D. Vandepitte, and W. Desmet. An efficient Wave Based Method for solving Helmholtz problems in three-dimensional bounded domains. Engineering Analysis with Boundary Elements, 36:63-75, 2012.

[47] E. Deckers, B. Bergen, B. Van Genechten, D. Vandepitte, and W. Desmet. An efficient Wave Based Method for 2D acoustic problems containing corner singularities. Computer Methods in Applied Mechanics and Engineering, 241-244:286-301, 2012.

[48] B. Pluymers, W. Desmet, D. Vandepitte, and P. Sas. On the use of a wave based prediction technique for steady-state structural-acoustic radiation analysis. Computer Modeling in Engineering $\mathcal{E}$ Sciences, 7(2):173-184, 2005.

[49] B. Bergen, B. Van Genechten, D. Vandepitte, and W. Desmet. An efficient Trefftz-based method for three-dimensional Helmholtz problems in unbounded domains. Computer Modeling in Engineering $\mathcal{G}$ Sciences, 61(2):155-175, 2010.

[50] B. Bergen, B. Pluymers, B. Van Genechten, D. Vandepitte, and W. Desmet. A Trefftz based method for solving Helmholtz problems in semi-infinite domains. Engineering Analysis with Boundary Elements, 36:30-38, 2012.

[51] C. Vanmaele, D. Vandepitte, and W. Desmet. An efficient wave based prediction technique for plate bending vibrations. Computer Methods in Applied Mechanics and Engineering, 196:3178-3189, 2007.

[52] C. Vanmaele, D. Vandepitte, and W. Desmet. An efficient wave based prediction technique for dynamic plate bending problems with corner stress singularities. Computer Methods in Applied Mechanics and Engineering, 198:2227-2245, 2009.

[53] C. Vanmaele, K. Vergote, D. Vandepitte, and W. Desmet. Simulation of in-plane vibrations of 2D structural solids with singularities using an efficient wave based prediction technique. Accepted for publication in Computer Assisted Mechanics and Engineering Sciences, 2012.

[54] H.L.F. Helmholtz. Über Integrale der hydrodynamischen Gleichungen, welch den Wirbelbewegungen entsprechen. Crelles Journal, 55:25$55,1958$.

[55] C. Vanmaele. Development of a wave based prediction technique for the efficient analysis of low-and mid-frequency structural vibrations. KULeuven, division PMA, PhD. thesis 2007D11, 2007.

[56] K. Vergote, C. Vanmaele, D. Vandepitte, and W. Desmet. An efficient wave based approach for the time-harmonic vibration analysis of 3D plate assemblies. Journal of Sound and Vibration, 332:1930-1946, 2013.

[57] B. Pluymers, W. Desmet, D. Vandepitte, and P. Sas. Application of an efficient wave-based prediction technique for the analysis of vibroacoustic radiation problems. J. Comput. Appl. Math., 168:353364, 2004.

[58] R. Lanoye, G. Vermeir, W. Lauriks, F. Sgard, and W. Desmet. Prediction of the sound field above a patchwork of absorbing materials. Journal of the Acoustical Society of America, 123:793-802, 2008.

[59] E. Deckers, N.-E. Hörlin, D. Vandepitte, and W. Desmet. A Wave Based Method for the efficient solution of the 2D poroelastic Biot equations. Computer Methods in Applied Mechanics and Engineering, 201-204:245-262, 2012.

[60] E. Deckers, D. Vandepitte, and W. Desmet. A Wave Based Method for the axisymmetric dynamic analysis of acoustic and poroelastic problems. Computer Methods in Applied Mechanics and Engineering, 257:1-16, 2013.

[61] E. Deckers, B. Van Genechten, D. Vandepitte, and W. Desmet. Efficient treatment of stress singularities in poroelastic Wave Based models using special purpose enrichment functions. Computers $\mathcal{E}$ Structures, 89:1117-1130, 2012.

[62] K. Vergote. Dynamic analysis of structural components in the mid frequency range using the wave based method, Non-determinism and inhomogeneities. KULeuven, division PMA, PhD. thesis 2012D03, 2012.

[63] B. van Hal, W. Desmet, D. Vandepitte, and P. Sas. A coupled finite elementwave based approach for the steady-state dynamic analysis of acoustic systems. J. Comput. Acoustics, 11(2):285-303, 2003.

[64] B. van Hal, W. Desmet, and D. Vandepitte. Hybrid finite element-wave-based method for steady-state interior structural-acoustic problems. Computers $\mathcal{E}$ Structures, 83(2-3):167-180, 2005

[65] J.B. Keller and D. Givoli. Exact non-reflecting boundary conditions. Journal of Computational Physics, 82:172-192, 1989.

[66] J.S. Lee, E. Deckers, S. Jonckheere, and W. Desmet. A direct hybrid wave based finite element modeling of poroelastic materials. In Proceedings of the Symposium on the Acoustics of Poro-Elastic Materials, SAPEM2011, Ferrara (Italy), 2011.

[67] S. Jonckheere and E. Deckers and B. Van Genechten and D. Vandepitte and W. Desmet. A direct hybrid Finite Element - Wave Based Method for the steady-state analysis of acoustic cavities with poro-elastic damping layers using the coupled Helmholtz-Biot equations. Computer Methods in Applied Mechanics and Engineering, 263:144-157, 2013. 
[68] P. Shorter and R. Langley. On the reciprocity relationship between direct field radiation and diffuse reverberant loading. Journal of the Acoustical Society of America, 117:85-95, 2005.

[69] K. Vergote, B. Van Genechten, D. Vandepitte, and W. Desmet. On the analysis of vibro-acoustic systems in the mid-frequency range using a hybrid deterministic-statistical approach. Computers $\mathcal{E}$ Structures, 89:868-877, 2011

[70] R. D'Amico, K. Vergote, R. Langley, and W. Desmet. On the use of the Lorentzian function for the evaluation of the frequency averaged input power into plates. In Proceedings of the 25th Conference on Noise and Vibration Engineering, ISMA2012, Leuven, (Belgium), 2012.

[71] K. Koo, B. Pluymers, W. Desmet, and S. Wang. Vibro-acoustic design sensitivity analysis using the wave-based method. Journal of Sound and Vibration, 330:4340-4351, 2011.

[72] S. Jonckheere and M. Vivolo and B. Pluymers and D. Vandepitte and W. Desmet. Vibro-acoustic characterisation of lightweight structures: A numerical-experimental approach. In Proceedings of the 7th International Styrian Nose, Vibration $\mathcal{F}$ Harshness Congress, Graz (Austria), 2012.

[73] O. Atak and D. Huybrechs and B. Pluymers and W. Desmet. The design of Helmholtz resonator based acoustic lenses by using the symmetric Multi-Level Wave Based Method and genetic algorithms. Submitted to Journal of Sound and Vibration, 2013. 